\title{
Uso de las Metodologías de Aprendizaje Colaborativo con TIC: Un análisis desde las creencias del profesorado
}

\author{
Rosalba Acosta Corporan \\ u139132@usal.es \\ Universidad de Salamanca, Spain \\ Antonio Víctor Martín-García \\ avmg@mail.com \\ Universidad de Salamanca, Spain
}

\section{Azucena Hernández Martín}

azuher@usal.es

Universidad de Salamanca, Spain

\begin{abstract}
Resumen
El objetivo principal del estudio es analizar las creencias de profesores de enseñanza media de la República Dominicana sobre la metodología basada en el aprendizaje colaborativo mediado por las TIC (CSCL). El CSCL es un modelo de enseñanza fundamentado en el compromiso mutuo de los participantes para alcanzar determinados objetivos educativos. Se trabajó con una muestra de 542 docentes a los que se aplicó un cuestionario adaptado, compuesto por 33 ítems que miden aspectos relacionados con el proceso de enseñanza y aprendizaje en entornos de uso de CSCL. Los resultados evidencian una valoración muy positiva sobre esta metodología por parte de los docentes para mejorar su desarrollo profesional. No perciben, sin embargo, tantos beneficios al emplearla con los estudiantes en la mejora del aprendizaje o del proceso de enseñanza en general. Se observan algunas diferencias entre los grupos en función de la variable años de experiencia docente. La sensibilización del profesorado sobre las ventajas de la metodología CSCL es una asignatura pendiente para la mejora de la calidad educativa; y ello exige impulsar debates internos en las instituciones escolares para promover el uso de esta metodología en los procesos de enseñanza y aprendizaje y en el desarrollo profesional docente.
\end{abstract}

\section{Palabras clave}

Aprendizaje Colaborativo; Innovación; Formación Continua; Metodología Didáctica; Profesorado 


\title{
Use of the Collaborative Learning Methodologies with ICT: An analysis based on the teachers' beliefs
}

\author{
Rosalba Acosta Corporan \\ u139132@usal.es \\ Universidad de Salamanca, Spain \\ Antonio Víctor Martín-García \\ avmg@mail.com \\ Universidad de Salamanca, Spain
}

\author{
Azucena Hernández Martín \\ azuher@usal.es \\ Universidad de Salamanca, Spain
}

\begin{abstract}
The main objective of this study is to analyze the beliefs of secondary or high school teachers of the Dominican Republic about the methodology based on collaborative learning by ICT (CSCL). The CSCL is a teaching model based on the mutual commitment of the participants to achieve certain educational objectives. We worked with a sample of 542 teachers to whom an adapted questionnaire was applied, consisting of 33 items that measure aspects related to the teaching and learning process in CSCL use environments. The results show a very positive assessment of this methodology by teachers to improve their professional development. They do not perceive, however, so many benefits when using it with students in the improvement of learning or the teaching process in general. Some differences between the groups are observed depending on the variable years of teaching experience. The sensitization of the teachers on the advantages of the CSCL methodology is a pending issue for the improvement of the educational quality; and this requires promoting internal debates in school institutions to promote the use of this methodology in teaching and learning processes and teacher professional development.
\end{abstract}

\section{Keywords}

Colaborative Learning; Innovation; Continuous Training; Didactic Methodology; Teacher 


\section{Introducción}

En las últimas décadas organismos internacionales como la OCDE, CEPAL, UNESCO, entre otros, han apostado por las Tecnologías de la Información y la Comunicación como factor esencial de cambio social mediante la mejora de procesos de formación tanto de estudiantes como docentes. Este hecho ha impulsado toda una serie de enfoques metodológicos, nuevas herramientas didácticas, como son las webs sociales, trabajo colaborativo a través de comunidades de aprendizaje y sistemas de gestión de cursos, uso educativo de blogs, wikis etc. (CEPAL, 2010, Gurría, 2016; UNESCO, 2013; Valencia-Molina et al., 2016). Muchos de estos enfoques están siendo aplicados en países con graves carencias sociales como son algunos países Latinoamericanos. Consciente de las debilidades que afectan gran parte de la población de estos países, la UNESCO ha establecido unos lineamientos materializados en una serie de metas educativas que deben ser cumplidas en el 2021. En su informe de 2013 la Unesco fijaba claramente el compromiso de los gobiernos de realizar una revolución educativa basada en las TIC (UNESCO, 2013).

Aunque estos esfuerzos institucionales se han incrementado en los últimos años, el informe PISA de 2015 deja muy mal parado a los países Latinoamericanos, en particular a la República Dominicana (RD), donde se obtuvieron los peores resultados en matemáticas de todos los países que conforman la OCDE. Dicho informe también reflejó que los docentes Iberoamericanos no utilizan con mucha frecuencia las TIC en el proceso de enseñanza aprendizaje. Esto condiciona las posibilidades de incorporar planteamientos innovadores en el aula (Gurría, 2016) y, sobre todo, limita la formación en competencias digitales de los estudiantes de cara a su posterior inserción laboral, así como, por extensión, el progreso y desarrollo social y económico de la región.

Las políticas educativas desplegadas por el Ministerio de Educación de RD intentan forzar la introducción de la innovación con entrenamientos en cursos de formación, capacitaciones no continuadas, dotación de equipamiento, etc., pero mantienen intocables los esquemas tradicionales de enseñanza, con escasas evidencias de estudios que aborden la concepción y creencias docentes sobre el uso de tecnologías digitales en sus prácticas docentes (vid. MINERD, 2013, 2014, 2015, 2016).

En este trabajo fijamos precisamente nuestra atención en analizar las creencias del profesorado sobre el uso de este tipo de planteamientos metodológicos, en particular, nos interesa conocer estas creencias sobre el denominado Aprendizaje Colaborativo Soportado por Computadoras o CSCL.

\section{Creencias del profesorado y Metodologías de Aprendizaje Colaborativo}

Son muchas y variadas las situaciones que condicionan el éxito de la labor docente, en su mayor parte relacionadas con el apoyo institucional, la práctica educativa y el desarrollo profesional (Quintero, 2008; OECD, 2016; Valencia-Molina et., 2016), así como con las concepciones y creencias del profesorado sobre determinados tipos de metodologías y la forma de utilizarlas. El CSCL es una de las metodologías docentes con mayor proyección en los últimos años, de hecho, consorcios internacionales, como el que elabora el NMC HORIZONT Report (2017), sitúan al aprendizaje colaborativo como una de las cinco tendencias mundiales que a corto plazo significará un avance en la adopción de nuevas tecnologías en educación. Esta tendencia hacia un mayor uso 
de la metodología CSCL se ha visto favorecida por el desarrollo y mejora de las plataformas de acceso abierto, al desarrollo del concepto de comunidades de aprendizaje o teorías como la conectividad y la cognición situada, implicando también la mejora y expansión de la formación continua y complementaria del profesorado en el uso de recursos y entornos virtuales (Burbules, 2014; Trujillo y Piretti, 2014).

El enfoque basado en CSCL ha pasado por diferentes concepciones, desde una visión marcadamente individualista, competitiva, unidireccional y mecánica a una redimensión más aceptada hoy basada en la cooperación y la colaboración grupal (Johnson y Johnson, 2009; Koschmann, 2012; Rubia y Guitert, 2014). De este modo, el CSCL se asocia con situaciones de enseñanza y de aprendizaje organizadas a través de actividades colaborativas, mediadas por computadoras, en las que interactúan grupos de estudiantes, docentes y otros actores educativos (Collazos, Jurado y Merchán, 2016).

Por eso, cuando aquí hablamos de metodologías de aprendizaje colaborativo con TIC nos referimos a una metodología basada en la negociación y construcción conjunta de saberes aplicable a todo el proceso de enseñanza-aprendizaje. Mediante este enfoque los alumnos diseñan su estructura de interacciones y mantienen el control sobre las diferentes decisiones que repercuten en su aprendizaje. Por su parte, el profesorado asume el rol que le corresponde como orientador y guía de ese proceso al tiempo que mejora sus competencias profesionales (Galindo et al., 2012).

Existe una abundante literatura especializada que describe las ventajas y beneficios atribuidos al $\mathrm{CSCL}$, desde facilitar la comunicación más allá del aula, fomentar la creación de redes colaborativas que operan de forma síncrona y asíncrona en la web, incrementar la motivación, mejorar la calidad de los aprendizajes al mejorar el clima de trabajo, desarrollar el aprendizaje mancomunado, optimizar el tiempo, crear interdependencia positiva, permite implicar dinámicas complejas entre los estudiantes, seguimiento docente, selección de buenas estrategias, evaluación continua, entre otras (Ceballos, 2005; Johnson y Johnson, 2009; Liu, Chen y Tai, (2017), etc.

A pesar de estos aspectos positivos, son muchos los centros docentes que utilizan esta metodología solo ocasionalmente y otros directamente no la emplean (García-Valcárcel, Basilotta y López, 2014). La relativamente escasa aplicación de este tipo de metodologías en determinados contextos está relacionada con diversos factores. Uno de ellos tiene que ver con la resistencia del profesorado a asumir nuevas tendencias, que se perciben con la exigencia de desarrollar procesos de cambio y esfuerzos de adaptación a los mismos (Cuadra y Romero 2003). También puede darse una falta de compromiso deontológico y de actualización técnica, o por una creencia negativa hacia ese enfoque (Sarramona, 2011). Esto último se refiere a que los profesores pueden no ser conscientes del impacto que generan estas metodologías en sus aulas. Por otro lado, se ha señalado también la ausencia de esfuerzos concertados, incapacidad cognitiva y actitudinal para adaptarse a los nuevos tiempos, falta de equipamiento, entre otros (Boza, Tirado y Guzmán-Franco (2010).

Se ha señalado también como factor explicativo el efecto del desgaste en la profesión. Así, el incremento de la presión social sobre el profesorado en general, las transformaciones tecnológicas y el incremento de trabajo que en la práctica docente implica su uso, la dificultad de cumplir temporalmente con los programas de contenidos, inconvenientes ligados a la distribución del tiempo, problemas con la evaluación, entre otros aspectos, aparecen como los protagonistas de este desgaste (Grace, 2013; Vaillant, 2006). Incluso se ha señalado que existe un alto índice de rechazo por parte del profesorado a integrar metodologías mediadas por las TIC porque tienden a considerar dichos métodos como una amenaza para su labor o incluso que pueden ser 
reemplazados por éstas en el futuro (Campos (2015). Mientras exista este alto índice de rechazo, el CSCL será más difícil de integrar en las prácticas docentes. La concepción docente solo se hará más positiva a medida que estos participen en procesos constructivistas emanados por las TIC (Ezquerra, Hamed, y Martín del Pozo, 2017).

Es evidente que esta resistencia a nuevos paradigmas educativos, sobre todo aquellos apoyados por las TIC, sigue siendo un problema bastante generalizado y un tema de discusión (Valdebenito y Durán, 2013; Mejía et al., 2018). Por este motivo resultan de interés los estudios que refuercen una línea de investigación que analice y contraste, en diferentes contextos geoculturales y socioeconómicos, las percepciones del profesorado sobre el uso de nuevas metodologías docentes.

En nuestro caso, hemos situado este estudio en un contexto geográfico y socioeconómico particular, como es la Republica Dominicana, que puede representar un ejemplo de país que deposita en el desarrollo tecnológico aplicado a la educación, grandes esperanzas de progreso social. Efectivamente, en la República Dominicana se ha producido en los últimos años un fuerte esfuerzo económico inversor en dotación tecnológica e infraestructura en las aulas, sin embargo, en la práctica el uso de estos recursos apenas son empleadas en el proceso de enseñanza aprendizaje (Coronado, Cantú y Rodríguez 2014; Román y Murillo 2014; Acosta, Martín y Hernández, 2017). A parte de las razones expuestas, existe una serie de factores que afectan las creencias de los docentes hacia el uso de metodologías digitales que pueden resumirse en dos tipos: externos e internos (Reyes y Martín-García, 2016). En cuanto a los factores externos tienen que ver con los equipamientos, disponibilidad de recursos, escasa formación docente, falta de disponibilidad de tiempo o de apoyo institucional, etc., mientras que los internos se refieren a la actitud docente, concepciones o creencias y resistencia al cambio (Brickner, 1995; Boza, Tirado y Guzmán-Franco, 2010).

\section{Método}

Como hemos indicado, la presente investigación se desarrolla en el contexto de República Dominicana, concretamente en las localidades de Santo Domingo, San Cristóbal y Villa Altagracia. Para la realización de este estudio se adopta un diseño de tipo cuantitativo, mediante la aplicación el cuestionario a centros educativos con equipamiento TIC. A continuación, se muestran los detalles de la población, modo de selección de la muestra, detalles del instrumento y procedimiento seguido para el análisis.

\section{a. Participantes}

La muestra participante en el estudio está compuesta por 542 profesores de enseñanza secundaria, divididos en dos grupos: profesores de Bachillerato ( $38 \%$ del total) y profesores de Institutos Técnico Profesional (62\%). Se realizó un muestreo de tipo intencional dadas las dificultades prácticas de aleatorizar la muestra. En todo caso, se buscaron centros de diferentes zonas de la República Dominica (un $57,7 \%$ en Santo Domingo, el $21,8 \%$ en San Cristóbal y un 20,5\% de Villa Altagracia) y características diversas que apoyaran la representatividad general de los resultados, contando finalmente con un número de 17 centros educativos participantes. De éstos, el $83 \%$ de carácter público y $17 \%$ de titularidad privada. El $78 \%$ están ubicados en el ámbito urbano, y un $22 \%$ en zona 
rural. En la muestra final participaron un $61 \%$ de profesoras y un $29 \%$ profesores, de ellos 114 profesores con más de 20 años de experiencia docente, 249 docentes entre 10 y 20 años y 179 con menos de 10 años de experiencia profesional. En cuanto al nivel de conocimiento de herramientas TIC el 64\% del profesorado indicó que tenía un nivel muy bajo, bajo o medio en el manejo de estos recursos, mientras a penas el $36 \%$ dijo poseer un nivel alto o muy alto.

\section{b. Instrumento}

Para la recogida de datos se utilizó un cuestionario elaborado por el grupo de investigación de la Universidad de Salamanca GITE y adaptado por nosotros para este estudio. El cuestionario fue utilizado previamente en una investigación aplicada en Castilla y León, donde se recogieron las percepciones y experiencias del profesorado sobre trabajo colaborativo con TIC en nivel de educación primaria y secundaria. En ese estudio se efectuaron pruebas de validez del mismo mediante el recurso de grupos de expertos (García-Varcárcel et al., 2015; Tejedor 2010), que avala su fundamentación teórica.

De este modo, el cuestionario utilizado está compuesto por 33 ítems distribuidos en 5 dimensiones o factores, todos ellos formulados en respuestas cerradas, con una escala tipo Likert de 5 puntos (donde uno significa totalmente en desacuerdo y cinco totalmente de acuerdo). La primera dimensión denominada "Práctica educativa" está conformada por los trece primeros ítems, y busca conocer las creencias generales del profesorado sobre el uso de metodologías aprendizaje colaborativo con TIC (CSCL) en sus prácticas docentes. Este factor se plantea en términos de ventajas de carácter general que tiene o puede tener el uso de este tipo de metodologías en el aula.

El resto de factores o dimensiones aluden a distintos efectos relacionados con la enseñanza y el aprendizaje en contextos CSCL que son valorados positiva o negativamente por los sujetos. Así, la segunda dimensión consta de 5 ítems para valorar en qué medida el factor "tiempo" es percibido como relevante en tanto que un factor negativo que limita el uso de $\mathrm{CSCL}$, señalado recurrentemente en la literatura especializada sobre el tema (Johnson y Johnson, 2009; Avello y Duart, 2016; Liu, Chen y Tai, 2017; Murcía, 2004; etc.). La tercera y cuarta dimensión se refieren a cuestiones sobre la percepción si las metodologías CSCL suponen "mejoras en la evaluación del alumnado" y/ mejoras en el "aprendizaje de los estudiantes". La quinta dimensión se refiere al grado de acuerdo o desacuerdo respecto a si el uso de este tipo de metodologías puede suponer una significativa mejora en el desarrollo profesional docente.

\section{c. Procedimiento}

Antes de aplicar el cuestionario fue contestado por seis docentes elegidos aleatoriamente de cada una de las regiones mencionadas anteriormente, quienes expresaron su conformidad respecto a la adecuada comprensión de cada uno de los ítems de la escala. Con este procedimiento nos aseguramos que la redacción del cuestionario estuviera contextualizado correctamente. Posteriormente, se accedió al Ministerio de Educación de República Dominicana (MINERD), concretamente al Departamento de Estadística, y se obtuvo un listado de los liceos o institutos que tenían recursos tecnológicos (TIC). Con estos datos y la autorización del Ministerio se procedió al muestreo, accediendo finalmente aquellos centros que mostraron su disponibilidad y facilidad de acceso. La aplicación del cuestionario se realizó por zonas y de forma presencial, para garantizar el máximo de respuestas y evitar la dispersión y reforzar la colaboración de los profesores de los centros previamente seleccionados. 
En cuanto al análisis de datos se optó por utilizar el software SPSS V21.

\section{Análisis y Resultados}

Una vez depurados los datos e invertido el sentido de algunos de los ítems para garantizar una adecuada interpretación estadística, se comenzó con un estudio de las características psicométricas de la escala. Aunque, como se ha indicado, teníamos información previa sobre el instrumento de recogida de datos, que garantizaba una adecuada validez de contenido, sin embargo, ésta no era suficiente para determinar la estructura factorial para nuestro contexto de aplicación. De este modo, el estudio de los ítems se realizó a partir de un análisis factorial exploratorio mediante el método de Ejes Principales para la extracción de factores, descartando el método de componentes principales, por la existencia de bastantes variables (ítems) con baja comunalidad (inferior a .60), así como también otros métodos que requieren supuesto de normalidad de las variables. La primera decisión al respecto fue calcular la medida KMO de Kaiser como medida de adecuación general de los datos obteniendo como valor .951 lo que muestra una muy satisfactoria matriz factorial (se valora .50 como valor de referencia medio). Se tuvieron en cuenta también como indicadores el índice de homogeneidad corregido (correlación ítem-total sin el ítem analizado) y el coeficiente alfa si se elimina el ítem de la escala (Lloret-Segura, Ferreres-Traver, Hernández Baeza y Tomás-Mardo, 2014). Finalmente se analizaron también los valores de dispersión y asimetría de los ítems.

El AFE mostró valores relativamente altos en 3 de los factores predeterminados inicialmente (Tabla 1), sin embargo el valor alpha de Cronbach para la consistencia interna de dos de las sub-escalas predefinidas está muy por debajo de lo deseable ( $\alpha=.677$ y .649 respectivamente), por lo que se decidió prescindir de las mismas en los análisis posteriores. Por lo tanto, definimos finalmente tres factores que identifican una serie de creencias sobre: F1:"Mejora de la práctica Educativa", F2:"Mejora del aprendizaje", F3:"Mejora del desarrollo profesional docente" que son percibidos por los sujetos a partir de la implementación de actividades que implican aprendizaje colaborativo en entornos mediados tecnológicamente.

Con respecto a la primera dimensión D1="Mejora de la Práctica Educativa", se observa que casi todas las medias están por encima de 4 puntos. La única excepción es el IT06, que se refiere a si "el trabajo colaborativo en el aula supone un cambio de cultura y una nueva concepción del proceso de enseñanza aprendizaje". No obstante. el mismo sigue siendo alto tomando en consideración que las escalas iban de 1 a 5 . En ese sentido, el $73 \%$ del profesorado manifestó estar de acuerdo o totalmente de acuerdo con este enunciado. En relación a las creencias sobre la influencia que tiene esta metodología en el aprendizaje de sus alumnos (D2="Mejora del aprendizaje"), más del 70\% de los docentes expresaron sentirse de acuerdo o muy de acuerdo en que la metodología basada en CSCL ofrece múltiples beneficios para fomentar el aprendizaje de sus estudiantes. Con respecto a la "mejora del desarrollo profesional" (D3) un elevado porcentaje expresaron que dicho modelo puede ser un cauce para desarrollar su creatividad y llevar a cabo nuevas ideas con el apoyo de otros compañeros. 


\begin{tabular}{|c|c|c|c|c|c|c|}
\hline $\begin{array}{c}\text { Factores o } \\
\text { Dimensiones } \\
\text { CSCL }\end{array}$ & $\begin{array}{l}\text { No } \\
\text { Ítems }\end{array}$ & $x$ & Sd. & $\begin{array}{l}\text { Correlación } \\
\text { total de } \\
\text { elementos } \\
\text { corregida }\end{array}$ & $\begin{array}{l}\text { Carga } \\
\text { Factorial }\end{array}$ & $\begin{array}{ll}\text { Alpha de } & \text { de } \\
\text { Cronbach } & \end{array}$ \\
\hline \multirow{6}{*}{$\begin{array}{l}\text { F1: Mejora de } \\
\text { la Práctica } \\
\text { Educativa }\end{array}$} & IT 01 & 4.28 & .796 & .562 & .661 & \multirow{12}{*}{$\alpha=.914$} \\
\hline & IT 02 & 4.32 & .760 & .612 & .729 & \\
\hline & IT 03 & 4.21 & .876 & .692 & .687 & \\
\hline & IT 04 & 4.32 & .802 & .722 & .710 & \\
\hline & IT 05 & 4.22 & .966 & .699 & .650 & \\
\hline & IT 06 & 3.99 & 1.060 & .579 & .538 & \\
\hline \multirow{6}{*}{$(n=542)$} & IT 07 & 4.25 & .801 & .645 & .630 & \\
\hline & IT 08 & 4.35 & .776 & .694 & .661 & \\
\hline & IT 09 & 4.24 & .839 & .653 & .703 & \\
\hline & IT 010 & 4.26 & .766 & .645 & .615 & \\
\hline & IT 011 & 4.14 & .857 & .662 & .657 & \\
\hline & IT 012 & 4.16 & .810 & .660 & .653 & \\
\hline \multirow{7}{*}{$\begin{array}{l}\text { F2: Mejora el } \\
\text { Aprendizaje } \\
\text { de } \\
\text { estudiantes }\end{array}$} & IT 23 & 4.08 & .986 & .681 & .688 & \multirow[t]{11}{*}{$\alpha=.847$} \\
\hline & IT 24 & 4.08 & .925 & .662 & .688 & \\
\hline & IT 25 & 3.71 & 1.070 & .487 & .458 & \\
\hline & IT 26 & 4.05 & .927 & .591 & .628 & \\
\hline & IT 27 & 4.33 & .723 & .646 & .655 & \\
\hline & IT 28 & 4.22 & .796 & .651 & .677 & \\
\hline & IT 29 & 3.94 & .977 & & .612 & \\
\hline \multirow{5}{*}{$\begin{array}{l}\text { F3: Mejora el } \\
\text { Desarrollo } \\
\text { Profesional } \\
\text { docente }\end{array}$} & IT 30 & 4.18 & .792 & .723 & .681 & \\
\hline & IT 31 & 4.26 & .893 & .753 & .751 & \\
\hline & IT 32 & 4.32 & .803 & .772 & .724 & \\
\hline & IT 33 & 74.22 & .949 & .739 & .753 & \\
\hline & & & & & & $\alpha=.882$ \\
\hline
\end{tabular}

Tabla 1. Análisis de ítems escalas

Fuente: Elaboración propia

Como hemos indicado, no hemos considerado en este análisis los factores $F=$ "Tiempo empleado en uso de CSCL" y $\mathrm{F}=$ "Mejora el proceso de Evaluación del Estudiante" que, aunque presentan porcentajes y medias de acuerdo también altas, agrupan algunos ítems con desviación típica por encima de 1 , que indica opiniones muy dispersas y en conjunto una baja consistencia interna.

Haciendo una valoración general, en cuanto al consenso y divergencia de las concepciones del profesorado la desviación típica indica que existe un mayor acuerdo por parte de los docentes al considerar que el empleo de estas actividades desarrolla la creatividad del alumnado y su capacidad de iniciativa (IT27= .723), ya que este es el ítem con menor dispersión de todo el cuestionario. Entre tanto, los datos apuntan que existe una mayor discrepancia en la creencia que el alumnado sea capaz de trabajar de forma autónoma y controlar su proceso de aprendizaje cuando realiza actividades colaborativas (IT25 $=1.070$ ). 
Tras hacer el análisis de la media ponderada que se muestra en la tabla 2 se observa que las medias mostraron que las cuestiones mejores valoradas por los docentes fueron F3=Desarrollo Profesional, seguida de F1=Práctica educativa" y F2="Aprendizaje.

\begin{tabular}{|l|r|r|r|r|r|}
\hline \multicolumn{1}{|c|}{ Dimensiones } & N & Mínimo & Máximo & Media & $\begin{array}{c}\text { Desviación } \\
\text { Estándar }\end{array}$ \\
\hline F1_Mejora Práctica Educativa & 542 & 1.00 & 5.00 & 4.23 & .603 \\
\hline F2_Mejora Aprendizaje & 542 & 1.14 & 5.00 & 4.06 & .665 \\
\hline F3_Mejora Desarrollo Prof. & 542 & 1.00 & 5.00 & 4.25 & .740 \\
\hline
\end{tabular}

Tabla 2. Factores o dimensiones creencias docentes sobre CSCL

Fuente: Elaboración propia

Por último, las tres dimensiones correlacionan positivamente entre sí (ver Tabla 3).

\begin{tabular}{|c|c|c|c|c|}
\hline & & $\begin{array}{l}\text { F1_media } \\
\text { ponderada }\end{array}$ & $\begin{array}{l}\text { F2_media } \\
\text { ponderada }\end{array}$ & $\begin{array}{l}\text { F3_media } \\
\text { ponderada }\end{array}$ \\
\hline \multirow[t]{3}{*}{$\begin{array}{l}\text { F1_"Mejora Práctica } \\
\text { Educativa" }\end{array}$} & $\begin{array}{l}\text { Correlación de } \\
\text { Pearson }\end{array}$ & 1 & $.707^{* *}$ & $.677^{* *}$ \\
\hline & Sig. (bilateral) & & $\mathrm{P}<.000$ & $\mathrm{P}<.000$ \\
\hline & $\mathrm{N}$ & 542 & 542 & 542 \\
\hline \multirow[t]{3}{*}{$\begin{array}{l}\text { F2_Mejora de } \\
\text { Aprendizaje }\end{array}$} & $\begin{array}{l}\text { Correlación de } \\
\text { Pearson }\end{array}$ & $.707^{* *}$ & 1 & $.738^{* *}$ \\
\hline & Sig. (bilateral) & $P<.000$ & & $P<.000$ \\
\hline & $\mathrm{N}$ & 542 & 542 & 542 \\
\hline \multirow[t]{3}{*}{$\begin{array}{l}\text { F3_Mejora Desarrollo } \\
\text { Profesional }\end{array}$} & $\begin{array}{l}\text { Correlación de } \\
\text { Pearson }\end{array}$ & $.677^{* *}$ & $.738^{* *}$ & 1 \\
\hline & Sig. (bilateral) & $\mathrm{P}<.000$ & $\mathrm{P}<.000$ & \\
\hline & N & 542 & 542 & 542 \\
\hline
\end{tabular}

Tabla 3. Correlación entre los factores analizados

Fuente: Elaboración propia

\section{a. Relación entre creencias del profesorado sobre CSCL y variables sociodemográficas y contextuales}

a.a. Diferencia de medias entre "Mejora de la práctica educativa" con CSCL y variables sociodemográficas. El análisis realizado en este punto revela que no existen diferencias significativas entre profesores y profesoras respecto a creencias sobre CSCL ni tampoco en la escala global. De igual modo, no aparecen diferencias entre los grupos en función del ámbito (rural o urbano), la titularidad del tipo de centro (público o privado) o la modalidad formativa del centro educativo (Bachillerato o FP). Si aparecen diferencias, sin embargo, respecto a la experiencia docente (medida en años de servicio docente). De este modo, los profesores con más de 20 años de servicio expresan peores valoraciones sobre las potenciales ventajas del uso de metodología CSCL que profesores que tienen una experiencia docente entre 10 y 20 años (ver tabla 4). 


\begin{tabular}{|c|c|c|c|}
\hline $\begin{array}{l}\text { Factor } \\
\text { CSCL }\end{array}$ & $\begin{array}{c}\text { Variables } \\
\text { Sociodemográfi } \\
\text { cas } \\
\text { y contextuales } \\
(n=542)\end{array}$ & $\begin{array}{c}\text { U de } \\
\text { Mann- whitney } \\
\text { P valor }\end{array}$ & $\begin{array}{c}\text { Contraste de } \\
\text { Hipótesis }\end{array}$ \\
\hline \multirow{5}{*}{$\begin{array}{l}\text { F1: } \\
\text { Mejora } \\
\text { Práctica } \\
\text { Educativa }\end{array}$} & Sexo & $\begin{array}{l}\text { U Mann- } \\
\text { whitney }=36.75 \\
\mathrm{P} \text { valor }=.286\end{array}$ & $\begin{array}{l}\text { Se acepta } \mathrm{H}_{\mathrm{O}} \text {. } \\
\text { No hay diferencias } \\
\text { entre los grupos }\end{array}$ \\
\hline & $\begin{array}{l}\text { Modalidad } \\
\text { Formativa } \\
\text { (FP/ Bachillerato) }\end{array}$ & $\begin{array}{l}\text { U Mann- } \\
\text { whitney }=34.71 \\
\text { p valor }=.861\end{array}$ & Se acepta $\mathrm{H}_{\mathrm{O}}$ \\
\hline & $\begin{array}{l}\text { Ámbito } \\
\text { (Rural/Urbano) }\end{array}$ & $\begin{array}{l}\text { U Mann- whitney= } \\
23.12 \\
\text { p valor }=.247\end{array}$ & Se acepta $\mathrm{H}_{\mathrm{O}}$ \\
\hline & $\begin{array}{l}\text { Experiencia } \\
\text { Docente (años) }\end{array}$ & $\begin{array}{l}\text { Kruskal-Wallis }= \\
10.35 \\
p \text { valor }=.006\end{array}$ & $\begin{array}{l}\text { Se acepta } \mathrm{H}_{1} \\
\text { (hay diferencias } \\
\text { entre los grupos) }\end{array}$ \\
\hline & $\begin{array}{l}\text { Tipo de Centro } \\
\text { (privado/público/ } \\
\text { subvencionado) }\end{array}$ & $\begin{array}{l}\text { Kruskal-Wallis }= \\
20.33 \\
\text { p valor }=.788\end{array}$ & Se acepta $\mathrm{H}_{\mathrm{O}}$ \\
\hline
\end{tabular}

Tabla 4. Relación Factor 1 y Variables sociodemográficas y contextuales

Fuente: Elaboración propia

a.b. Diferencia de medias entre "Mejora del aprendizaje" con CSCL y variables sociodemográficas. Los resultados en este apartado son muy similares a los del F1, únicamente aparecen diferencias significativas entre los sujetos en la creencia que la metodología CSCL mejora el aprendizaje de los alumnos en relación con la experiencia docente (entre profesores de más de 20 años y el resto de profesores), mostrando aquellos puntuaciones más bajas en la escala (análisis post doc: diferencias entre "Más de 20 " y menos de 10 ,p=.025 y "más de 20" y de "10 a 20", p=.015 ) (ver tabla 5).

\begin{tabular}{|c|c|c|c|}
\hline $\begin{array}{l}\text { Factor } \\
\text { CSCL }\end{array}$ & $\begin{array}{c}\text { Variables } \\
\text { Sociodemográfi } \\
\text { cas } \\
\text { y contextuales } \\
(n=542)\end{array}$ & $\begin{array}{c}\text { U de } \\
\text { Mann- whitney } \\
\text { P valor }\end{array}$ & $\begin{array}{c}\text { Contraste de } \\
\text { Hipótesis }\end{array}$ \\
\hline \multirow{3}{*}{$\begin{array}{l}\text { F2: Mejora } \\
\text { Aprendizaj } \\
\text { e de los } \\
\text { Estudiantes }\end{array}$} & Sexo & $\begin{array}{l}\text { U Mann- } \\
\text { whitney }=34.503 \\
P \text { valor }=.840\end{array}$ & Se acepta $\mathrm{H}_{\mathrm{O}}$ \\
\hline & $\begin{array}{l}\text { Modalidad } \\
\text { Formativa } \\
\text { (FP/ Bachillerato) }\end{array}$ & $\begin{array}{l}\text { U Mann- } \\
\text { whitney }=37.56 \\
\text { p valor }=.073\end{array}$ & Se acepta $\mathrm{H}_{\mathrm{O}}$ \\
\hline & $\begin{array}{l}\text { Ámbito } \\
\text { (Rural/Urbano) }\end{array}$ & $\begin{array}{l}\text { U Mann- whitney }= \\
\text { 21.94. } p \text { valor }=.051\end{array}$ & Se acepta $\mathrm{H}_{\mathrm{O}}$ \\
\hline
\end{tabular}




\begin{tabular}{|l|l|l|l|}
\hline & $\begin{array}{l}\text { Experiencia } \\
\text { Docente (años) }\end{array}$ & $\begin{array}{l}\text { Kruskal-Wallis=9.06. } \\
\text { p valor=.011 }\end{array}$ & Se acepta $\mathrm{H}_{1}$ \\
\cline { 2 - 4 } & $\begin{array}{l}\text { Tipo de Centro } \\
\text { (privado/público/ } \\
\text { subvencionado) }\end{array}$ & $\begin{array}{l}\text { Kruskal-Wallis }=21.82 \\
\text { p valor }=.412\end{array}$ & Se acepta $\mathrm{H}_{\mathrm{O}}$ \\
\hline
\end{tabular}

Tabla 5. Relación Factor 2 y Variables sociodemográficas y contextuales

Fuente: Elaboración propia

a.c. Diferencia de medias entre "Mejora del desarrollo profesional" con CSCL y variables sociodemográficas. No aparecen diferencias entre hombres y mujeres respecto a este factor. Tampoco respecto al tipo de centro (público o privado). Sí en cambio los profesores que trabajan en centros de modalidad bachillerato valoran más positivamente la metodología CSCL para la mejora del desarrollo profesional docente que los que lo hacen en centros de formación técnica-profesional. Asimismo, lo hacen en el mismo sentido los profesores de centros ubicados en entorno rural. Por último, cuanto menos años de experiencia mejores valoraciones en este factor (ver Tabla 6)

\begin{tabular}{|c|c|c|c|}
\hline $\begin{array}{l}\text { Factor } \\
\text { CSCL }\end{array}$ & $\begin{array}{c}\text { Variables } \\
\text { Socio- } \\
\text { demográficas } \\
\text { y contextuales } \\
(n=542)\end{array}$ & $\begin{array}{l}\text { U de } \\
\text { Mann- whitney } \\
\text { P valor }\end{array}$ & $\begin{array}{l}\text { Contraste } \\
\text { de } \\
\text { Hipótesis }\end{array}$ \\
\hline \multirow{5}{*}{$\begin{array}{l}\text { F3: Mejora } \\
\text { Desarrollo } \\
\text { profesiona } \\
\text { I }\end{array}$} & Sexo & $\begin{array}{l}\text { U Mann- whitney }=34.39 \\
\text { P valor }=.792\end{array}$ & $\begin{array}{l}\text { Se acepta } \\
\mathrm{H}_{\mathrm{O}}\end{array}$ \\
\hline & $\begin{array}{l}\text { Modalidad } \\
\text { Formativa } \\
\text { (FP/ Bachillerato) }\end{array}$ & $\begin{array}{l}\text { U Mann- whitney }=38.78 \\
\text { p valor }=.012\end{array}$ & $\begin{array}{l}\text { Se acepta } \\
\mathrm{H}_{1}\end{array}$ \\
\hline & $\begin{array}{l}\text { Ámbito } \\
\text { (Rural/Urbano) }\end{array}$ & $\begin{array}{l}\text { U Mann- whitney= } \\
20.74 \\
\text { p valor }=.006\end{array}$ & $\begin{array}{l}\text { Se acepta } \\
\mathrm{H}_{1}\end{array}$ \\
\hline & $\begin{array}{l}\text { Experiencia } \\
\text { Docente (años) }\end{array}$ & $\begin{array}{l}\text { Kruskal-Wallis }=25.15 \\
\mathrm{p} \text { valor }=.000\end{array}$ & $\begin{array}{l}\text { Se acepta } \\
\mathrm{H}_{1}\end{array}$ \\
\hline & $\begin{array}{l}\text { Tipo de Centro } \\
\text { (privado/público/ } \\
\text { subvencionado) }\end{array}$ & $\begin{array}{l}\text { Kruskal-Wallis }=21.04 \\
\text { p valor }=.799\end{array}$ & $\begin{array}{l}\text { Se acepta } \\
\mathrm{H}_{\mathrm{O}}\end{array}$ \\
\hline
\end{tabular}

Tabla 6. Relación Factor 3 y Variables sociodemográficas y contextuales Fuente: Elaboración propia

a.d. Diferencia de medias entre "Escala general CSCL y variables sociodemográficas. Únicamente se observan diferencias entre los grupos en cuanto a la experiencia docente (Tabla 7) ("Mas de 20 años" y "menos de 10 " $(\mathrm{p}=.026)$ y "más de 20 años" y de 10 a 20 años $(\mathrm{p}=.001)$

\begin{tabular}{|l|l|l|l|}
\hline & Variables & U de & Contraste \\
\hline
\end{tabular}




\begin{tabular}{|c|c|c|c|}
\hline $\begin{array}{l}\text { Factor } \\
\text { CSCL }\end{array}$ & $\begin{array}{c}\text { Sociodemográfic } \\
\text { as } \\
\text { y contextuales } \\
(n=542)\end{array}$ & $\begin{array}{l}\text { Mann- whitney } \\
\text { P valor }\end{array}$ & $\begin{array}{l}\text { de } \\
\text { Hipótesis }\end{array}$ \\
\hline \multirow[t]{5}{*}{$\begin{array}{l}\text { Escala } \\
\text { Global }\end{array}$} & Sexo & $\begin{array}{l}\text { U Mann- } \\
\text { whitney }=36.75 \\
P \text { valor }=.605\end{array}$ & Se acepta $\mathrm{H}_{\mathrm{O}}$ \\
\hline & $\begin{array}{l}\text { Modalidad } \\
\text { Formativa } \\
\text { (FP/ Bachillerato) }\end{array}$ & $\begin{array}{l}\text { U Mann- whitney }= \\
36.36 \\
p \text { valor }=.267\end{array}$ & Se acepta $\mathrm{H}_{\mathrm{O}}$ \\
\hline & $\begin{array}{l}\text { Ámbito } \\
\text { (Rural/Urbano) }\end{array}$ & $\begin{array}{l}\text { U Mann- } \\
\text { whitney }=22.18 \\
P \text { valor }=.074\end{array}$ & Se acepta $\mathrm{H}_{\mathrm{o}}$ \\
\hline & $\begin{array}{l}\text { Experiencia } \\
\text { Docente (años) }\end{array}$ & $\begin{array}{l}\text { Kruskal- Wallis }=12.52 \\
\mathrm{p} \text { valor }=.002\end{array}$ & Se acepta $\mathrm{H}_{1}$ \\
\hline & $\begin{array}{l}\text { Tipo de Centro } \\
\text { (público/privado) }\end{array}$ & $\begin{array}{l}\text { Kruskal-Wallis }=21.33 \\
\mathrm{p} \text { valor }=.642\end{array}$ & Se acepta $\mathrm{H}_{\mathrm{O}}$ \\
\hline
\end{tabular}

Tabla 7. Relación ESCALA CSCL y Variables sociodemográficas y contextuales Fuente: Elaboración propia

\section{Discusión y Conclusiones}

Después de haber realizado este estudio se ha llegado a las siguientes conclusiones. Primero, se comprueba que el profesorado de República Dominicana participante en el estudio tiene una valoración general positiva sobre el uso de Metodologías de Aprendizaje Colaborativo mediadas por las TIC, ya que la mayor parte de los ítems obtuvieron puntuaciones por encima de la media de la escala. Este hallazgo coincide con lo expuesto por Cabezas. Casillas y Hernández (2016) quienes sostienen que los docentes identifican más ventajas que inconvenientes de este modelo de enseñanza. En esa línea, dentro de las dimensiones analizadas la que presenta una más alta valoración es la que apunta a ventajas que recaen sobre el propio desarrollo profesional docente, y no tanto sobre beneficios sobre los estudiantes, ya sea en la mejora del aprendizaje o la mejora del proceso de enseñanza en general. De alguna manera puede inferirse que al mejorar la competencia de los profesores al usar nuevas metodologías docentes como son las basadas en $\mathrm{CSCL}$, hay un efecto también positivo en la mejora del proceso de aprendizaje de los estudiantes. Pero este efecto se ve también limitado por aspectos como puede ser tiempo invertido o dificultades de evaluación de los estudiantes cuando se usa CSCL, que pueden pesar sobre la valoración de esta metodología (Campos, 2015). Aunque no han sido analizadas explícitamente en este estudio, hemos señalado que más del $70 \%$ de la muestra sostuvo que las actividades colaborativas requieren mucho tiempo de dedicación para el profesorado y el alumnado y el $48 \%$ expresó estar de acuerdo o muy de acuerdo que es difícil evaluar a cada estudiante o saber lo que ha aprendido con el empleo de dichas metodologías. 
En segundo lugar, las creencias de los profesores sobre CSCL en función del tipo y características de los centros en los que trabajan (centros públicos o privados) o el ámbito (rural, urbano) apenas muestran diferencias importantes. Destaca una valoración más positiva de profesores de ámbitos rurales en las posibilidades de CSCL para su actualización y la innovación docente, que aparece también en algunos estudios realizados al respecto (Pérez Reinoso, 2016; Sarramona, 2011).

Tercero, un resultado claro de nuestro trabajo tiene que ver con los años de experiencia docente $y$, en gran medida relacionada con la misma, también con la edad de los sujetos. Así, hemos apreciado diferencias entre los profesores en el sentido que los de menor edad y menos años de ejercicio profesional valoran de manera más positiva las ventajas y beneficios del uso de aprendizaje colaborativo en contextos de formación virtual. Concretamente, en relación al uso de la metodología en el aula, desarrollo del aprendizaje y desarrollo profesional. En parte este resultado concuerda con lo planteado por autores como Grace (2013) y Vaillant (2006), quienes señalan que a mayor experiencia profesional se van fijando patrones de trabajo que correlacionan con menor compromiso de actualización y menos motivación hacia el uso de nuevas tendencias o innovaciones educativas. Una conclusión evidente de este tipo de resultados lleva a enfatizar la necesidad de enfocar de manera particular acciones de formación e información directamente dirigidas a profesores más veteranos, de manera que ayuden a conocer mejor estas metodologías $y$, en su caso, modificar las creencias sobre las mismas.

Por todo lo expuesto consideramos importante concienciar al profesorado sobre las ventajas de la metodología CSCL, tanto para la mejora de la calidad educativa, como en su formación permanente (Rubia y Guitert, 2014). En ese sentido, coincidimos con Boza, Tirado y Guzmán-Franco (2010), sobre el papel de las administraciones en el impulso de debates internos en las comunidades escolares y centros educativos que estimulen su uso, arbitrando medidas de apoyo para el desarrollo profesional. Esto implica la formación en competencias que permitan una adecuada implementación lo que podrá modificar y mejorar su concepción sobre las mismas (Noguera, Guerrero-Roldan y Maso, 2018).

Por último, y en cuanto a las limitaciones del estudio, aunque un aspecto positivo a valorar es el tamaño de la muestra final participante en nuestro estudio (542 docentes), y el contar con la autorización y apoyo del Ministerio de Educación de República Dominica, las dificultades prácticas (época de exámenes, falta de tiempo, falta de implicación de algunos centros, etc.) impidieron una mejor selección muestral, que hubiera permitido el concurso de más centros educativos para mejorar el poder de generalización de los resultados. Por este motivo, para futuras investigaciones sería interesante llegar a una mayor población de docentes introduciendo también un enfoque cualitativo en la recogida de datos.

\section{Referencias}

Acosta, R., Martín, A., y Hernández, A. (2017). Uso de metodologías de aprendizaje colaborativo con TIC: un reto para el profesorado de Bachillerato de Rep. Dominicana. En I Congreso Virtual Internacional y III Congreso Virtual Iberoamericano sobre Recursos Educativos Innovadores (CIREI, 2017), 314.

Avello, R., y Duart, J. M. (2016). Nuevas tendencias de aprendizaje colaborativo en e-learning: Claves para su implementación efectiva. Estudios pedagógicos, 42 (1), 271-282. 
Boza, Á., Tirado, R., y Guzmán-Franco, M.D (2010). Creencias del profesorado sobre el significado de la tecnología en la enseñanza: influencia para su inserción en los centros docentes andaluces. RELIEVE, 16 (1). Recuperado de: https://www.uv.es/RELIEVE/v16n1/RELIEVEv16n1_5

Brickner, D. L. (1995). The effects of first and second order barriers to change on the degree and nature of computer usage of mathematics teachers: A case study. Dissertation Abstracts International, 56 (1), 07A. (UMI No. 9824700).

Burbules, N. C. (2014). El aprendizaje ubicuo: nuevos contextos, nuevos procesos. Entramados: educación y sociedad, 1(1), 131-135.

Cabezas, M., Casillas, S., y Hernández , A. (2016). Metodologías de trabajo colaborativo en la Educación Secundaria. RELATEC/Revista Latinoamericana de Tecnología Educativa, 15 (1), 76-85.

Campos, J.A. (2015). Lo cotidiano (entre usos y resistencias) de las tic en un aula de la educación secundaria obligatoria. Apuntes etnográficos. Revista de Antropología Experimental, 15, 567-580.

Ceballos, F.S. (2005). Diez razones para trabajar en red. Colombia: Diálogo cultural y tecnologías de información y comunicación para el fortalecimiento de los procesos comunitarios. Colombia.

CEPAL (2010). Panorama Social en América Latina. Cap. 2. La educación frente a la reproducción de la desigualdad y la exclusión: Situación y desafíos en América Latina. Recuperado de: https://www.cepal.org/es/publicaciones/1236-panorama-social-america-latina-2010

Collazos, C. A., Jurado, J. L., y Merchán, L. (2016). Entornos ubicuos y colaborativos (U-CSCL) para ambientes de enseñanza-aprendizaje de competencias profesionales. Cali: Bonaventuriana.

Coronado Cabrera, E., Cantú Valadez, M., y Rodríguez Pichardo, C. (2014). Diagnóstico universitario sobre el uso de la TIC en el proceso de enseñanza-aprendizaje bajo la modalidad educativa presencial en Santo Domingo. Edutec. Revista Electrónica de Tecnología Educativa, 50, 1-14. doi: http://dx.doi.org/10.21556/edutec.2014.50.225

Cuadra, G., y Romero, R. (2003). Concepciones y creencias del profesorado de secundaria sobre enseñanza y aprendizaje de las matemáticas. Enseñanza de la Ciencia, 21 (1), 27-47.

Ezquerra, A., Hamed, S., y Martín del Pozo, R. (2017). El cambio de las concepciones en futuros maestros sobre los contenidos escolares de ciencias. Revista Complutense de Educación, 28 (3), 779-796.

Galindo González, R., Galindo González, L., Martínez de la Cruz, N., Ley Fuentes, M., Ruiz Aguirre, E., y Valenzuela González, E. (2013). Acercamiento epistemológico a la teoría del aprendizaje colaborativo. Apertura, 4 (2), 156-169.

García-Valcárcel, A. (2015). Proyectos de trabajo colaborativo con TIC. Madrid: Síntesis.

García-Valcárcel, A., Basilotta, V., y López, C. (2014). ICT in Collaborative Learning in the Classrooms of Primary and Secondary Education. [Las TIC en el aprendizaje colaborativo en el aula de Primaria y Secundaria]. Comunicar, 42, 65-74. https://doi.org/10.3916/C42-2014-06

Grace, L. (2013). La construcción de una identidad docente, ¿Un desafío para la política educativa?. Revista Exitus, 3 (1), 75-87.

Gurría, A. (2016). PISA 2015: resultados Claves. París: OECD

Johnson, D. W., y Johnson, R. T. (2009). An educational psychology success story: Social interdependence theory and cooperative learning. Educacional Researcher, 38, 365-379. doi: https://dx.doi.org/10.3102/0013189x09339057

Koschmann, T. (2012). CSCL: Theory and practice of an emerging paradigm. New York: Routledge.

Lloret-Segura. S., Ferreres-Traver. A., Hernández-Baeza. A., y Tomás-Marco. I. (2014). El Análisis Factorial Exploratorio de los Ítems: una guía práctica. revisada y actualizada. Anales de Psicología, 30 (3), 11511169.

Mejía, A. J., Villarreal, C., Silva, C., Suarez-Suarez, D., y Villamizar Niño, C. (2018). Estudio de los factores de resistencia al cambio y actitud hacia el uso educativo de las TIC por parte del personal docente. Revista Boletín Redipe, 7 (2), 53-63. 
Ministerio de Educación de Republica Dominicana MINERD. Memorias institucionales. Recuperado de: http://www.ministeriodeeducacion.gob.do/sobre-nosotros/memorias

Murcia, E. P. C. (2004). Aprendizaje colaborativo soportado por computador (CSCL): su estado actual. Revista Iberoamericana de Educación, 33 (6), 1-15.

NMC Horizon Report: 2017 Higher Education Edition. The New Media Consortium and the Educause Learning Initiative. Recuperado de: http://cdn.nmc.org/media/2017-nmc-horizon-report-he-EN.pdf

Noguera, I., Guerrero-Roldán, A. E., y Masó, R. (2018). Collaborative agile learning in online environments: Strategies for improving team regulation and project management. Computers \& Education, 116, 110129.

OECD (2016). PISA 2015 Results (Volume II) Policies and Practices for Successful Schools. doi: http://dx.doi.org/10.1787/9789264267510-en

Quintero, A. (2008). Innovación educativa e integración curricular de las TIC. En García-Valcárcel (Coord.). Investigación y tecnologías de la información y comunicación al servicio de la innovación educativa (pp. 9-32). Salamanca: Aquilafuente.

Reyes-González, D., y Martín-García, A.V. (2016). Creencias de docentes en formación que afectan el uso de recursos tecnológicos. Ciencia, Docencia y Tecnología, 27 (53), 293-314.

Rubia, B. y Guitert, M. (2014). Revolution in Education: Computer Support for Collaborative Learning. [¿La revolución de la enseñanza? El aprendizaje colaborativo en entornos virtuales (CSCL)]. Comunicar, 42, 10-14. https://doi.org/10.3916/C42-2014-a2

Sarramona, J. (2011). ¿Qué Significa ser profesional docente en la actualidad?. Revista Portuguesa de Pedagogía, 427-440.

Tejedor, F, J, (Coord.) (2010). Evaluación de procesos de innovación escolar basados en el uso de las TIC desarrollados en la comunidad de Castilla y León. Salamanca: Aquilafuente.

Trujillo, C. C., y Pieretti, M. R. (2014). Computer-supported collaborative learning (CSCL): buenas prácticas educativas en la universidad. In Gonzálvez Vallés (Coord.). Nuevas tendencias en innovación educativa superior (pp. 141-162). ACCI (Asociación Cultural y Científica Iberoamericana).

UNESCO (2013). Situación Educativa de América Latina y el Caribe: Hacia la educación de calidad para todos al 2015. Santiago: Unesco.org

Vaillant, D. (2006). Atraer y retener buenos profesionales en la profesión docente: políticas en Latinoamérica. Revista de educación, 340 (1), 117-140.

Valencia-Molina, T., Serna, A., Ochoa, S., Caicedo, A. M., Montes, J. A., y Chavez, J. D. (2016). Competencias y estándares TIC desde la dimensión pedagógica: Una perspectiva desde los niveles de apropiación de las TIC en la práctica educativa docente. Cali: Pontificia Universidad Javeriana y UNESCO. 